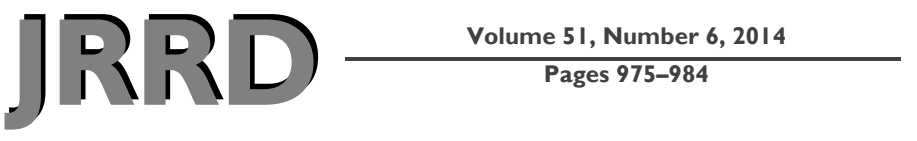

\title{
Dynamometer-based measure of spasticity confirms limited association between plantarflexor spasticity and walking function in persons with multiple sclerosis
}

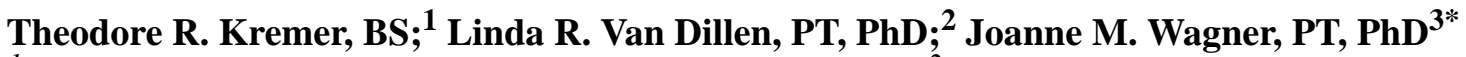 \\ ${ }^{1}$ School of Medicine, Saint Louis University, St. Louis, MO; ${ }^{2}$ Program in Physical Therapy, Washington University \\ School of Medicine, St. Louis, MO; ${ }^{3}$ Department of Physical Therapy and Athletic Training, Doisy College of Health \\ Sciences, Saint Louis University, St. Louis, MO
}

\begin{abstract}
The literature shows inconsistent evidence regarding the association between clinically assessed plantarflexor (PF) spasticity and walking function in ambulatory persons with multiple sclerosis (pwMS). The use of a dynamometerbased spasticity measure (DSM) may help to clarify this association. Our cohort included 42 pwMS (27 female, 15 male; age: $42.9+/-10.2$ yr) with mild clinical disability (Expanded Disability Status Scale score: $3.6+/-1.6)$. PF spasticity was assessed using a clinical measure, the modified Ashworth Scale (MAS), and an instrumented measure, the DSM. Walking function was assessed by the timed 25-foot walk test (T25FWT), the 6-minute walk test (6MWT), and the 12-item Multiple Sclerosis Walking Scale (MSWS-12). Spearman rho correlations were used to evaluate relationships between spasticity measures, measures of walking speed and endurance, and self-perceived limitations in walking. The correlation was small between PF spasticity and the T25FWT (PF maximum [Max] MAS rho $=0.27$, PF Max DSM rho $=0.26$ ), the 6MWT (PF Max MAS rho $=-0.20$, PF Max DSM rho $=-0.21$ ), and the MSWS-12 (PF Max MAS rho $=0.11$, PF Max DSM rho = 0.26). Our results are similar to reports in other neurologic clinical populations, wherein spasticity has a limited association with walking dysfunction.
\end{abstract}

Key words: 12-item Multiple Sclerosis Walking Scale, ambulation, dynamometer, modified Ashworth Scale, multiple sclerosis, self-perceived limitations, spasticity, walking dysfunction, walking endurance, walking speed.

\section{INTRODUCTION}

Multiple sclerosis (MS) is a chronic, inflammatory disease of the central nervous system (CNS) characterized by demyelination and loss of motor and sensory axons within the brain and spinal cord [1]. Spasticity, defined as a motor disorder characterized by a velocitydependent increase in tonic stretch reflexes and increased tendon jerks resulting from disinhibition of the stretch reflex [2], is a typical consequence of pathological changes within the corticospinal system. Lower-limb spasticity is believed to be a key contributor to mobility disability in persons with MS (pwMS). As such, lowerlimb spasticity is routinely assessed in clinical practice to

\footnotetext{
Abbreviations: 6MWT $=6$-minute walk test, $\mathrm{CNS}=$ central nervous system, DF = dorsiflexion, DSM = dynamometerbased spasticity measure, EDSS = Expanded Disability Status Scale, EMG = electromyography, MAS = modified Ashworth Scale, Max = maximum, MS = multiple sclerosis, MSWS-12 = 12-item Multiple Sclerosis Walking Scale, PF = plantarflexor, pwMS = persons with multiple sclerosis, $\mathrm{ROM}=$ range of motion, RRMS = relapsing-remitting multiple sclerosis, T25FWT = timed 25-foot walk test.

*Address all correspondence to Joanne M. Wagner, PT, PhD; Program in Physical Therapy, 3437 Caroline Mall, Suite 1026, St. Louis, MO 63104; 314-977-8532; fax: 314977-8513. Email: jwagne34@slu.edu http://dx.doi.org/10.1682/JRRD.2013.08.0186
} 
document clinical disability and monitor disease progression, and therapies aimed at reducing spasticity are employed to promote better walking in pwMS [3-4].

PwMS perceive spasticity to be related to selfreported mobility disability [5]. Nonetheless, there is inconsistent evidence regarding the association between lower-limb spasticity, measured using the modified Ashworth Scale (MAS) [6], and walking speed, walking endurance, and self-perceived walking limitations. Sosnoff et al. reported on a group of 34 pwMS, 15 of whom exhibited plantarflexor (PF) spasticity [7]. In this cohort of pwMS, the group with PF spasticity had slower walking speed $(p=0.02)$, reduced walking endurance ( $p<$ 0.01 ), and greater self-perceived walking limitations ( $p=$ 0.04 ) than the group without spasticity. In contrast, we recently reported a small relationship between PF spasticity measured using the MAS and walking speed $(\rho=$ $0.27, p=0.08)$, walking endurance $(\rho=-0.20, p=0.20)$, and self-perceived walking limitations $(\rho=0.11, p=$ 0.48 ) [8]. There also were no differences in walking speed $(p=0.34)$, walking endurance $(p=0.72)$, or selfperceived walking limitations $(p=0.64)$ in pwMS with PF MAS scores greater than $0(n=29)$ compared with pwMS with PF MAS equal to $0(n=13)$.

The MAS is the most commonly reported clinical measure of spasticity; however, the objectivity of the MAS has been questioned [9]. Dynamometer-based spasticity measures (DSMs), which objectively quantify velocity-dependent resistance to passive movement, have been developed to address the subjectivity of the MAS [10-14]. Dynamometer-based estimates of lower-limb spasticity have confirmed a limited association between lower-limb spasticity, as measured using clinical scales, and walking dysfunction in other neurologic clinical populations [10-14]. Quantifying spasticity using a DSM in addition to the MAS may clarify the ambiguous evidence regarding the effect of lower-limb spasticity on walking dysfunction in pwMS. A better understanding of the association between lower-limb spasticity and walking dysfunction in pwMS is required for the development of targeted therapeutic interventions aimed at improving walking.

The purpose of this study was to determine the effect of PF spasticity on walking function in pwMS by examining the relationships between clinically assessed PF spasticity; a DSM of PF spasticity; and walking function assessed by standardized measures of walking speed, walking endurance, and self-perceived walking limita- tions. We hypothesized based on our previous work in this cohort [8], and based on studies of walking dysfunction in people with other CNS conditions, that there is a limited association between PF spasticity, whether measured clinically or using a dynamometer, and walking speed, walking endurance, and self-perceived walking limitations in pwMS.

\section{METHODS}

\section{Participants}

Study participants included 45 ambulatory pwMS. All participants were recruited through the Multiple Sclerosis Clinic at Saint Louis University, The John L. Trotter Multiple Sclerosis Clinic at Washington University School of Medicine, and the Gateway chapter of the National Multiple Sclerosis Society. Participants were included (1) if they had a diagnosis of MS from a boardcertified neurologist, including relapsing-remitting MS (RRMS), secondary progressive MS, and primary progressive MS; (2) if they were 18 to 65 yr old; (3) if they had minimal to moderate clinical disability as evidenced by an Expanded Disability Status Scale (EDSS) score ranging from 0.0 to 6.0 , as determined by a boardcertified neurologist; and (4) if they had RRMS but were at least 6 mo following their last clinical exacerbation of MS. Participants were excluded if they (1) had lowerlimb orthopedic conditions that limited ambulation, (2) were pregnant, or (3) were unable to provide informed consent.

\section{Data Collection}

PF spasticity was measured bilaterally using the MAS [6]. The MAS provides a rating of spasticity on a 0 to 4 scale, where $0=$ no increase in muscle tone and $4=$ affected part(s) are rigid in flexion or extension. The MAS has been found to be a reliable measure in pwMS [15].

Walking function was assessed by standardized measures of walking speed, walking endurance, and self-perceived walking limitations. The timed 25 -foot walk test (T25FWT), a component of the Multiple Sclerosis Functional Composite, is a standardized clinical measure of short-distance maximal walking speed [16]. The 6-minute walk test (6MWT) is a standardized clinical measure of walking endurance [17]. The 12-item Multiple Sclerosis Walking Scale (MSWS-12) is a self-report measure of the effect of MS on walking ability [18]. The MSWS-12 
provides a score from 0 to 100 , with larger values indicating a greater perception of walking difficulty due to MS. These tests were administered following standardized procedures by a physical therapist.

PF spasticity was also quantified using a Biodex System 4 dynamometer (Biodex Medical Systems, Inc; Shirley, New York). Both ankles were tested in a random order. Each participant was placed on the dynamometer in a semisupine position. For the tested limb, the leg was positioned so that the knee was in full extension and supported by the dynamometer chair. The foot was placed in the dynamometer dorsiflexion (DF)/plantar flexion attachment, and the ankle joint center was aligned with the center of rotation of the dynamometer. All participants were tested wearing shoes. Ankle range of motion (ROM) limits were preestablished to allow for a total of $40^{\circ}$ of ROM, ranging from $30^{\circ} \mathrm{PF}$ to $10^{\circ} \mathrm{DF}$. Waist and knee straps were used to stabilize the pelvis and lower limb, and a foot rest provided support for the nontested limb. For the test, the ankle was passively rotated from PF to DF 3 times at an angular velocity of $5 \%$ s and 10 times at angular velocities of 20,60, and $90 \%$ [19]. Participants were instructed not to help move the lever arm and remain relaxed while spasticity measurements were being taken. DSMs have moderate reliability [10,12]. Bipolar surface electromyography (EMG) electrodes (MA300 EMG system, Motion Lab Systems Inc; Baton Rouge, Louisiana) placed on the gastroc-soleus and anterior tibialis were used to visually monitor agonist and antagonist muscle activity, respectively, to ensure the movement was passive. Analog torque (foot-pounds), position (degrees), and velocity (degrees per second) signals were sampled directly from the dynamometer at a rate of $1 \mathrm{kHz}$ using a PowerLab 16/30 (ADInstruments; Dunedin, New Zealand) and LabChart Pro version 7 software (ADInstruments) and saved directly to disk for subsequent analysis.

\section{Data Analysis}

Because the MAS includes a score of $1+$, the raw PF MAS scores were transformed to a 0 to 5 scale [20]. The PF maximum (Max) MAS score (PF Max MAS) for each participant was determined and used for subsequent analysis. PwMS with PF MAS scores 1.0 or more for either leg were classified as having spasticity, whereas pwMS with PF MAS scores less than 1.0 on both legs were classified as not having spasticity [7].
For the DSM, data analysis was performed using LabChart Pro version 7 and MATLAB R2011b (The MathWorks Inc; Natick, Massachusetts). Torque angle data were low-pass filtered $(50 \mathrm{~Hz})$ then processed to correct for the effects of limb weight and gravity using an anthropomorphic method [21]. The weight of the foot was assumed to be 1.45 percent of the entire body weight [22]. The first trial of each velocity was excluded from analyses. To determine the average resistance to stretch for an entire trial, the work done on the dynamometer was calculated by determining the area under the gravitycorrected torque versus angle curve. To exclude end effects caused by the dynamometer, the boundaries of the area were set at $-5^{\circ},-30^{\circ}$, the torque curve, and the zero torque line. Work can be calculated using the formula Equation 1:

$$
\int_{-5^{\circ}}^{-30^{\circ}} T \times d \theta
$$

where $T=$ the torque produced against the dynamometer and $d \theta=$ a small change in the angle (Figure 1) [10]. The

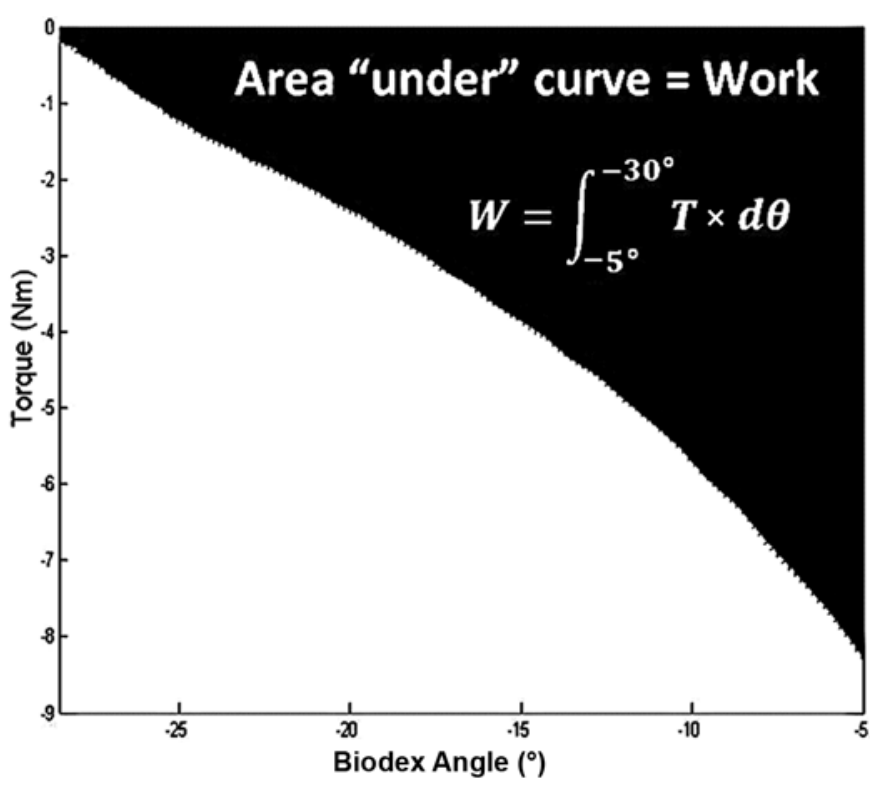

Figure 1.

Work was calculated by determining area between gravitycorrected torque versus angle curve and zero torque. To exclude end effects, boundaries of area were set at $-5^{\circ},-30^{\circ}$, torque curve, and zero torque line. Work can be calculated using the equation, where $T=$ torque produced against dynamometer and $d \theta=$ small change in angle. 
average work was calculated for each individual at each velocity tested. Linear regression was used to determine the slope of the line of best fit for the average work as a function of velocity (Figure 2). This slope has been shown to be a quantitative measure of spasticity in that it represents a velocity-dependent resistance to passive stretch [10]. The maximum slope value calculated for each participant was used for subsequent analysis (PF Max DSM).

\section{Statistical Analysis}

Statistical analyses were performed in SPSS version 20 (IBM Corporation; Armonk, New York). Significance was accepted at $p<0.05$. Since distributions were not nor-

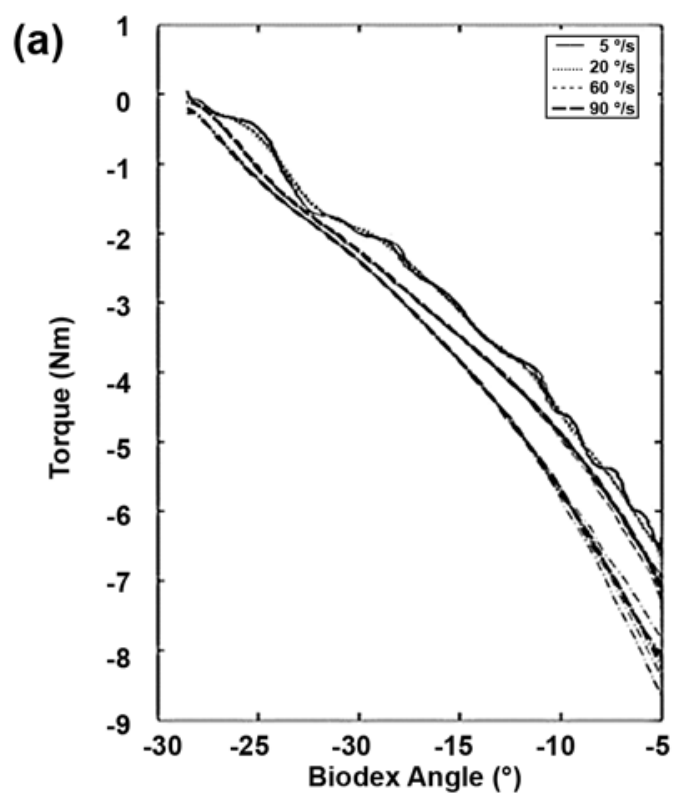

(b)
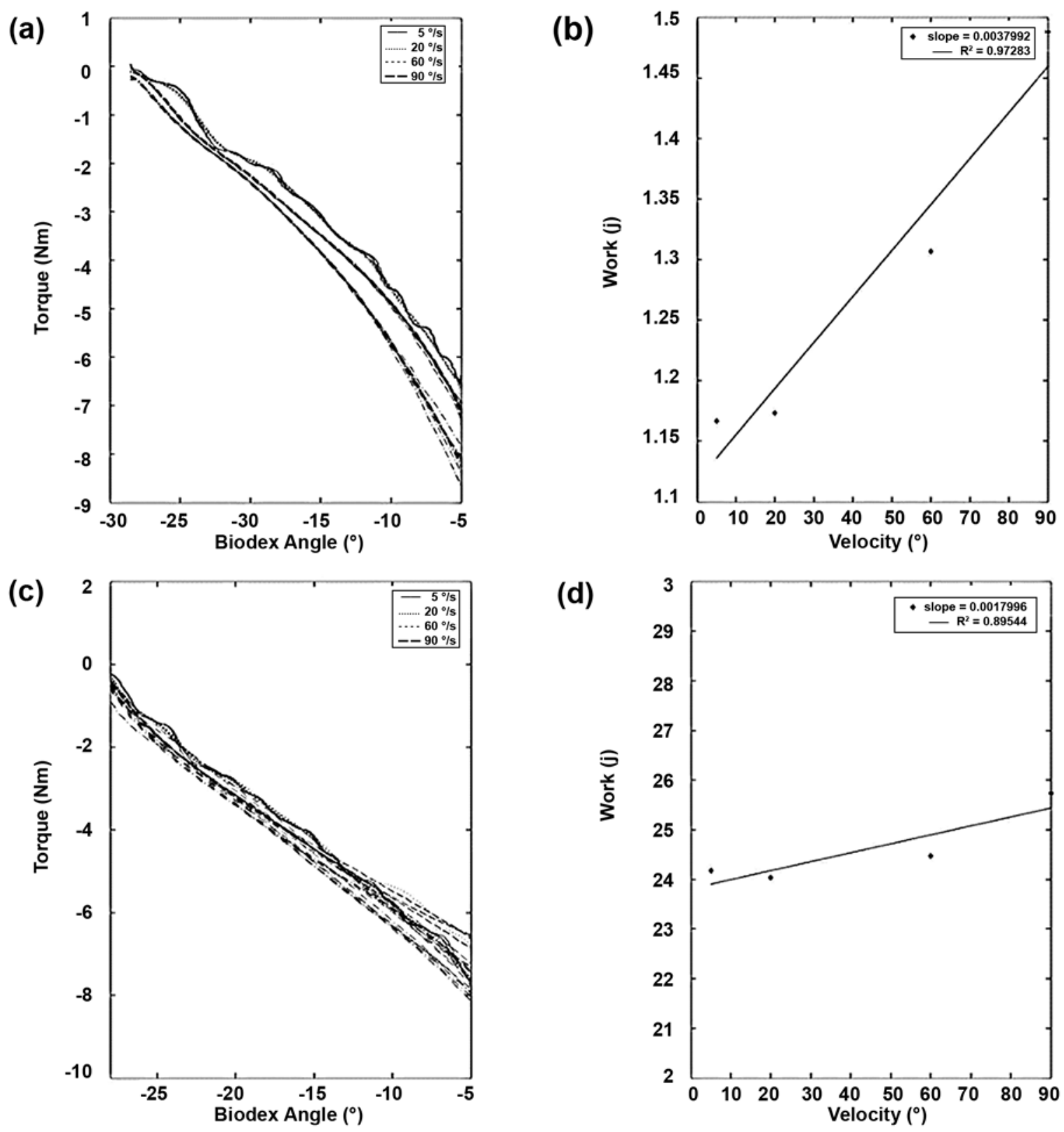

Figure 2.

Raw data for person with multiple sclerosis (a) with clinically assessed spasticity (plantarflexor modified Ashworth Scale [PF MAS] = 3) at different velocities, (b) with associated regression line of best fit, (c) with raw data without spasticity (PF MAS = 0) at different velocities, and (d) with associated regression line of best fit. 
mal, Spearman rho correlations were used to evaluate relationships between spasticity measures (PF Max MAS, PF Max DSM) and measures of walking speed (T25FWT), walking endurance (6MWT), and self-perceived limitations in walking (MSWS-12). Interpretation of the magnitude of the correlation coefficients were as follows: weak $(r<0.30)$, moderate $(0.30 \leq r<0.70)$, and strong $(r \geq 0.70)$ [23]. Based on our sample size, correlation coefficients greater than 0.30 and 0.49 were statistically significant at the $p<0.05$ and $p<0.001$ levels, respectively [24]. Mann-Whitney U tests assessed differences in DSM between pwMS with a MAS $\geq 1$ and pwMS with a MAS $=0$. The magnitude of the difference between groups (pwMS with a MAS $\geq 1$ vs pwMS with a MAS $=0$ ) was estimated by calculating the Cohen $d$ effect size [25].

\section{RESULTS}

Three participants were excluded from data analysis because their dynamometer data could not be processed: one participant due to an extremely limited DF ROM and two participants because the ankle was unable to be passively rotated due to ankle clonus or leg spasms confirmed by visual inspection of EMG. Table 1 provides demographics and clinical characteristics of the remaining participants $(n=42)$. Based on median EDSS scores, our sample of pwMS had mild clinical disability [26]. Of the participants, 12 (29\%) reported using a straight cane for community ambulation. Seven (17\%) pwMS used a cane during the T25FWT. Nine (21\%) pwMS used a cane during the 6MWT. Three of these participants wore a unilateral ankle-foot orthosis during testing. Six (14\%) pwMS reported the use of oral antispasticity medications.

Our sample of pwMS had mild PF spasticity based on the PF Max MAS and PF Max DSM values (Table 2) [27]. Of the participants, 69 percent (29/42) were classified as having clinically assessed spasticity based on a PF MAS score $\geq 1$ on either leg. The PF Max MAS and the PF Max DSM were measured in the same limb in 90 percent (38/42) of participants. PF Max DSM values did not differ between pwMS with a MAS $\geq 1$ and pwMS with a MAS $=0(0.0040 \pm 0.0015$ vs $0.0037 \pm 0.0012, p=0.38$, $d=0.23)$. There was a weak relationship between the clinically assessed measures and DSMs of PF spasticity (PF Max MAS vs PF Max DSM: $\rho=0.260, p=0.10$ ).
Table 1.

Participant demographics.

\begin{tabular}{lc}
\hline \multicolumn{1}{c}{ Variable } & Value \\
\hline Participants $(n)$ & 42 \\
Age (yr), mean \pm SD (range) & $42.9 \pm 10.2(24-63)$ \\
BMI, mean \pm SD (range) & $26.7 \pm 5.4(20.4-42.7)$ \\
Female, $n(\%)$ & $27(64)$ \\
Race, $n(\%)$ & \\
Caucasian & $30(71)$ \\
African American & $12(29)$ \\
EDSS, median (IQR) [range] & $3.0(2.9-4.8)[0-6]$ \\
Type of MS, $n$ (\%) & $35(83)$ \\
RR & $5(12)$ \\
SP & $2(5)$ \\
PP & $7.7 \pm 6.1(1-20)$ \\
Time Since Diagnosis (yr), \\
$\quad$ mean \pm SD (range)
\end{tabular}

Our sample of pwMS had mild to moderate limitations in walking speed and endurance $[17,28]$ and self-perceived limitations in walking (Table 2). Regardless of the method, PF spasticity weakly correlated with the T25FWT (PF Max MAS: $\rho=0.27, p=0.08$; PF Max DSM: $\rho=$ 0.26, $p=0.10$ ), 6MWT (PF Max MAS: $\rho=-0.20, p=$ 0.20 ; PF Max DSM: $\rho=-0.21, p=0.18$ ), and MSWS12 (PF Max MAS: $\rho=0.11, p=0.48$; PF Max DSM: $\rho=0.26$, $p=0.09)$.

\section{DISCUSSION}

The major finding of this study is that PF spasticity has a limited association with walking speed, walking endurance, and self-perceived walking limitations in pwMS with mild clinical disability, regardless of whether spasticity was measured clinically or using a dynamometer. The DSM confirmed our previous report of a weak association between clinically assessed PF spasticity and walking dysfunction in this cohort of pwMS [8]. Our results are consistent with reports in other neurologic clinical populations, wherein spasticity quantified with clinical scales or instrumented measures has a limited association with walking dysfunction [29-32]. The poor association found between PF spasticity and walking dysfunction may help explain the limited efficacy of spasticity management for improving walking in pwMS [33-34], at least in pwMS with mild spasticity and clinical disability. 
JRRD, Volume 51, Number 6, 2014

Table 2.

Spasticity and walking measures for persons with multiple sclerosis $(n=42)$.

\begin{tabular}{lccc}
\hline \multicolumn{1}{c}{ Measure } & Mean & Standard Deviation & Range \\
\hline PF Max MAS (score) & 1.43 & 1.33 & $0-4$ \\
PF Max DSM (J/[\%/s]) & 0.0039 & 0.0014 & $0.0022-0.0096$ \\
T25FWT (s) & 5.84 & 2.30 & $3.68-14.8$ \\
6MWT (m) & 466.7 & 133.9 & $157.9-677.0$ \\
MSWS-12 (score) & 42.9 & 22.3 & $0-90.5$ \\
& Median & Interquartile Range & Range \\
PF Max MAS (score) & 1 & $0-2$ & $0-4$ \\
6MWT = 6-minute walk test, DSM = dynamometer-based spasticity measure, MAS = modified Ashworth Scale, Max = maximum, MSWS-12 = 12-item Multiple \\
Sclerosis Walking Scale, PF = plantarflexor, T25FWT = timed 25-foot walk test.
\end{tabular}

Self-reported walking difficulties become more common and more pronounced in pwMS as the severity of self-reported spasticity increases [5,35]. It is possible that the weak association found between PF spasticity and self-reported walking dysfunction in our cohort of pwMS is due to the low levels of spasticity in our cohort of pwMS. A stronger association might exist between PF spasticity, quantified using the MAS or DSM, and selfreported walking dysfunction in pwMS with more severe PF spasticity or in pwMS exhibiting clonus.

Our results differ from a report on pwMS where those with PF spasticity were found to have greater mobility impairment than those without PF spasticity [7]. In Sosnoff et al., the spasticity group had considerably greater clinical disability than the no spasticity group [7]. The association between PF spasticity and mobility impairment was not reported. In our cohort, clinical disability was similar between the spasticity and no spasticity groups. We have previously reported no differences in walking between the groups [8]. Because the level of PF spasticity was similar in both studies, it is possible that additional impairments contributing to overall disability (i.e., weakness, sensory loss, and ataxia) may have contributed to the group differences in mobility reported by Sosnoff et al. [7].

The weak association between the MAS and DSM in our cohort of ambulatory pwMS is congruent with reports in other neurologic clinical populations, demonstrating a limited association between clinical and instrumented measures of upper- and lower-limb spasticity [10,13-14,36]. Collectively, these results suggest that the MAS and DSM may quantify different components of spasticity. The MAS quantifies "increase in muscle tone," which may be due to both reflex and nonreflex components of resistance to passive movement [6]. The
DSM quantifies the change in work at different velocities. The nonreflex component (e.g., stiffness) of resistance to passive movement should be similar at different velocities. Therefore, we believe the DSM primarily quantifies the reflex component of resistance to passive stretch. Our study design does not allow us to determine the unique contribution of the nonreflex component of spasticity [37]. Differences in participant positioning for the two measures may also contribute to the weak association between clinical and instrumented measures. Additionally, the limited range of MAS scores in our cohort of ambulatory pwMS may have attenuated the relationship between the MAS and DSM [14].

The median MAS score for the spasticity group was slightly larger than the median MAS score for the no spasticity group. Consistent with the MAS, the average DSM value for pwMS with clinically assessed PF spasticity was slightly larger than the value for pwMS without clinically assessed PF spasticity. The DSM values for pwMS with a MAS $\geq 1$ and pwMS with a MAS $=0$ were similar to the PF DSM value reported for children without disability [8]. Thus, the DSM confirmed the mild severity of clinically assessed PF spasticity in our cohort of ambulatory pwMS with mild disability.

The current study has limitations. First, pwMS in this investigation had low levels of clinical spasticity; therefore, the results may not generalize to ambulatory pwMS with more pronounced clinical spasticity. Second, pwMS exhibiting clonus or muscle spasms during the DSM were excluded from analyses because it invalidated our algorithm for processing velocity-dependent resistance to passive stretch. Third, the ROM during the DSM was truncated due to the deceleration artifact of the dynamometer. Thus, the DSM did not evaluate spasticity over the entire ROM. Fourth, because reflexes depend on the 
specific task and limb positioning [38], spasticity measured in the semisupine position may not be equivalent to spasticity measured during ambulation. Fifth, spasticity was only evaluated at the ankle; we are unable to determine whether, and to what extent, spasticity of other muscle groups contribute to walking dysfunction in pwMS. Finally, our study was not designed to investigate the contribution of other lower-limb impairments to walking dysfunction.

\section{CONCLUSIONS}

PF spasticity has a limited association with walking speed, walking endurance, and self-perceived walking limitations in pwMS with mild clinical disability, regardless of whether spasticity was measured clinically using the MAS or using a dynamometer. The weak association between the DSM and the MAS suggests that the two tests may quantify different components of spasticity.

\section{ACKNOWLEDGMENTS}

\section{Author Contributions:}

Study concept and design: L. R. Van Dillen, J. M. Wagner.

Acquisition of data: J. M. Wagner.

Analysis and interpretation of data: T. R. Kremer, J. M. Wagner.

Drafting of manuscript: T. R. Kremer, L. R. Van Dillen, J. M. Wagner.

Final approval of manuscript: T. R. Kremer, L. R. Van Dillen,

J. M. Wagner.

Obtained funding: J. M. Wagner.

Financial Disclosures: Dr. Wagner serves on the speaker's bureau of Acorda Therapeutics Inc, Ardsley, New York.

Funding/Support: This material was based on work supported by the National Institutes of Health (grant K12 HD055931, primary investigator: J. M. Wagner).

Additional Contributions: We thank Anne H. Cross, MD, for her assistance with participant recruitment. We thank all participants for their involvement in the study; Elissa Held Bradford, MSPT, NCS, MSCS, for assistance with data collection; and the Gateway chapter of the National Multiple Sclerosis Society for assistance with recruitment of participants.

Institutional Review: The study was approved by the Saint Louis University Institutional Review Board and the Washington University Human Research Protection Office, and all participants provided informed consent prior to participation.

Participant Follow-Up: The authors have no plans to notify the study subjects of the publication of this article because approval to contact participants about publication was not included in the informed consent process.

\section{REFERENCES}

1. Lassmann H. The pathology of multiple sclerosis and its evolution. Philos Trans R Soc Lond B Biol Sci. 1999; 354(1390):1635-40. [PMID:10603616] http://dx.doi.org/10.1098/rstb.1999.0508

2. Lance JW. Symposium synopsis: Spasticity disordered motor control. Chicago (IL): Year Book; 1980. p. 485-94.

3. Orsnes GB, Sørensen PS, Larsen TK, Ravnborg M. Effect of baclofen on gait in spastic MS patients. Acta Neurol Scand. 2000;101(4):244-48. [PMID:10770520]

4. Amatya B, Khan F, La Mantia L, Demetrios M, Wade DT. Non pharmacological interventions for spasticity in multiple sclerosis. Cochrane Database Syst Rev. 2013;2: CD009974. [PMID:23450612]

5. Rizzo MA, Hadjimichael OC, Preiningerova J, Vollmer TL. Prevalence and treatment of spasticity reported by multiple sclerosis patients. Mult Scler. 2004;10(5):589-95. [PMID:15471378] http://dx.doi.org/10.1191/1352458504ms1085oa

6. Bohannon RW, Smith MB. Interrater reliability of a modified Ashworth scale of muscle spasticity. Phys Ther. 1987; 67(2):206-7. [PMID:3809245]

7. Sosnoff JJ, Gappmaier E, Frame A, Motl RW. Influence of spasticity on mobility and balance in persons with multiple sclerosis. J Neurol Phys Ther. 2011;35(3):129-32. [PMID:21934374] http://dx.doi.org/10.1097/NPT.0b013e31822a8c40

8. Wagner JM, Kremer TR, Van Dillen LR, Naismith RT. Plantarflexor weakness negatively impacts walking in persons with multiple sclerosis more than plantarflexor spasticity. Arch Phys Med Rehabil. 2014;95(7):1358-65. [PMID:24582617] http://dx.doi.org/10.1016/j.apmr.2014.01.030

9. Johnson GR. Outcome measures of spasticity. Eur J Neurol. 2002;9(Suppl 1):10-16, 53-61. [PMID:11918644] http://dx.doi.org/10.1046/j.1468-1331.2002.0090s1010.x

10. Engsberg JR, Olree KS, Ross SA, Park TS. Quantitative clinical measure of spasticity in children with cerebral palsy. Arch Phys Med Rehabil. 1996;77(6):594-99. [PMID:8831478] http://dx.doi.org/10.1016/S0003-9993(96)90301-9

11. Biering-Sørensen F, Nielsen JB, Klinge K. Spasticityassessment: A review. Spinal Cord. 2006;44(12):708-22. [PMID:16636687] http://dx.doi.org/10.1038/sj.sc.3101928

12. Pierce SR, Lauer RT, Shewokis PA, Rubertone JA, Orlin MN. Test-retest reliability of isokinetic dynamometry for the assessment of spasticity of the knee flexors and knee extensors in children with cerebral palsy. Arch Phys Med Rehabil. 2006;87(5):697-702. [PMID:16635633] http://dx.doi.org/10.1016/j.apmr.2006.01.020 
13. Pandyan AD, Price CI, Rodgers H, Barnes MP, Johnson GR. Biomechanical examination of a commonly used measure of spasticity. Clin Biomech (Bristol, Avon). 2001; 16(10):859-65. [PMID:11733123]

http://dx.doi.org/10.1016/S0268-0033(01)00084-5

14. Pandyan AD, Price CI, Barnes MP, Johnson GR. A biomechanical investigation into the validity of the modified Ashworth Scale as a measure of elbow spasticity. Clin Rehabil. 2003;17(3):290-93. [PMID:12735536] http://dx.doi.org/10.1191/0269215503cr610oa

15. Ghotbi N, Nakhostin Ansari N, Naghdi S, Hasson S. Measurement of lower-limb muscle spasticity: Intrarater reliability of Modified Modified Ashworth Scale. J Rehabil Res Dev. 2011;48(1):83-88. [PMID:21328165] http://dx.doi.org/10.1682/JRRD.2010.02.0020

16. Cutter GR, Baier ML, Rudick RA, Cookfair DL, Fischer JS, Petkau J, Syndulko K, Weinshenker BG, Antel JP, Confavreux C, Ellison GW, Lublin F, Miller AE, Rao SM, Reingold S, Thompson A, Willoughby E. Development of a multiple sclerosis functional composite as a clinical trial outcome measure. Brain. 1999;122(5):871-82.

[PMID:10355672]

http://dx.doi.org/10.1093/brain/122.5.871

17. Goldman MD, Marrie RA, Cohen JA. Evaluation of the sixminute walk in multiple sclerosis subjects and healthy controls. Mult Scler. 2008;14(3):383-90. [PMID:17942508] http://dx.doi.org/10.1177/1352458507082607

18. Hobart JC, Riazi A, Lamping DL, Fitzpatrick R, Thompson AJ. Measuring the impact of MS on walking ability: the 12-Item MS Walking Scale (MSWS-12). Neurology. 2003;60(1):31-36. [PMID:12525714] http://dx.doi.org/10.1212/WNL.60.1.31

19. Ross SA, Engsberg JR. Relation between spasticity and strength in individuals with spastic diplegic cerebral palsy. Dev Med Child Neurol. 2002;44(3):148-57. [PMID:12005315]

20. Wagner JM, Lang CE, Sahrmann SA, Hu Q, Bastian AJ, Edwards DF, Dromerick AW. Relationships between sensorimotor impairments and reaching deficits in acute hemiparesis. Neurorehabil Neural Repair. 2006;20(3):406-16. [PMID:16885427] http://dx.doi.org/10.1177/1545968306286957

21. Kellis E, Baltzopoulos V. Gravitational moment correction in isokinetic dynamometry using anthropometric data. Med Sci Sports Exerc. 1996;28(7):900-907. [PMID:8832545] http://dx.doi.org/10.1097/00005768-199607000-00018

22. Winter DA. Biomechanics of human movement. 1st ed. New York (NY): Wiley; 1979.

23. Gerstman BB. Basic biostatistics: Statistics for public health practice. Sudbury (MA): Jones and Bartlett Publishers; 2008.
24. Rosenthal R, Rosnow RL. Essentials of behavioral research: Methods and data analysis. New York (NY): McGraw-Hill; 1984.

25. Cohen J. Statistical power analysis for the behavioral sciences. 2nd ed. Hillsdale (NJ): L. Erlbaum Associates; 1988.

26. Feys P, Gijbels D, Romberg A, Santoyo C, Gebara B, de Noordhout BM, Knuts K, Béthoux F, de Groot V, Vaney C, Dalgas U. Effect of time of day on walking capacity and self-reported fatigue in persons with multiple sclerosis: A multi-center trial. Mult Scler. 2012;18(3):351-57.

[PMID:21969239]

http://dx.doi.org/10.1177/1352458511419881

27. Engsberg JR, Ross SA, Olree KS, Park TS. Ankle spasticity and strength in children with spastic diplegic cerebral palsy. Dev Med Child Neurol. 2000;42(1):42-47.

[PMID:10665974]

28. Gijbels D, Dalgas U, Romberg A, de Groot V, Bethoux F, Vaney C, Gebara B, Medina CS, Maamâgi H, Rasova K, de Noordhout BM, Knuts K, Feys P. Which walking capacity tests to use in multiple sclerosis? A multicentre study providing the basis for a core set. Mult Scler. 2012;18(3):364-71.

[PMID:21952098] http://dx.doi.org/10.1177/1352458511420598

29. Ada L, Vattanasilp W, O’Dwyer NJ, Crosbie J. Does spasticity contribute to walking dysfunction after stroke? J Neurol Neurosurg Psychiatry. 1998;64(5):628-35. [PMID:9598679] http://dx.doi.org/10.1136/jnnp.64.5.628

30. Nadeau S, Arsenault AB, Gravel D, Bourbonnais D. Analysis of the clinical factors determining natural and maximal gait speeds in adults with a stroke. Am J Phys Med Rehabil. 1999;78(2):123-30. [PMID:10088586] http://dx.doi.org/10.1097/00002060-199903000-00007

31. Ng SS, Hui-Chan CW. Contribution of ankle dorsiflexor strength to walking endurance in people with spastic hemiplegia after stroke. Arch Phys Med Rehabil. 2012;93(6): 1046-51. [PMID:22440486] http://dx.doi.org/10.1016/j.apmr.2011.12.016

32. Ross SA, Engsberg JR. Relationships between spasticity, strength, gait, and the GMFM-66 in persons with spastic diplegia cerebral palsy. Arch Phys Med Rehabil. 2007; 88(9):1114-20. [PMID:17826455] http://dx.doi.org/10.1016/j.apmr.2007.06.011

33. Arroyo R, Vila C, Clissold S. Retrospective observational study of the management of multiple sclerosis patients with resistant spasticity in Spain: the '5E' study. Expert Rev Pharmacoecon Outcomes Res. 2011;11(2):205-13. [PMID:21476822] http://dx.doi.org/10.1586/erp.11.6

34. Barnes MP, Kent RM, Semlyen JK, McMullen KM. Spasticity in multiple sclerosis. Neurorehabil Neural Repair. 
2003;17(1):66-70. [PMID:12645447]

http://dx.doi.org/10.1177/0888439002250449

35. Oreja-Guevara C, González-Segura D, Vila C. Spasticity in multiple sclerosis: Results of a patient survey. Int J Neurosci. 2013;123(6):400-408. [PMID:23297730] http://dx.doi.org/10.3109/00207454.2012.762364

36. Pierce SR, Johnston TE, Shewokis PA, Lauer RT. Examination of spasticity of the knee flexors and knee extensors using isokinetic dynamometry with electromyography and clinical scales in children with spinal cord injury. J Spinal Cord Med. 2008;31(2):208-14. [PMID:18581670]

37. Lorentzen J, Grey MJ, Crone C, Mazevet D, BieringSørensen F, Nielsen JB. Distinguishing active from passive components of ankle plantar flexor stiffness in stroke, spinal cord injury and multiple sclerosis. Clin Neurophysiol. 2010;121(11):1939-51. [PMID:20457538]

http://dx.doi.org/10.1016/j.clinph.2010.02.167

38. Kakebeeke TH, Lechner H, Baumberger M, Denoth J, Michel D, Knecht H. The importance of posture on the isokinetic assessment of spasticity. Spinal Cord. 2002;40(5):
236-43. [PMID:11987006]

http://dx.doi.org/10.1038/sj.sc.3101282

Submitted for publication August 30, 2013. Accepted in revised form March 18, 2014.

This article and any supplementary material should be cited as follows:

Kremer TR, Van Dillen LR, Wagner JM. Dynamometerbased measure of spasticity confirms limited association between plantarflexor spasticity and walking function in persons with multiple sclerosis. J Rehabil Res Dev. 2014; 51(6):975-84.

http://dx.doi.org/10.1682/JRRD.2013.08.0186

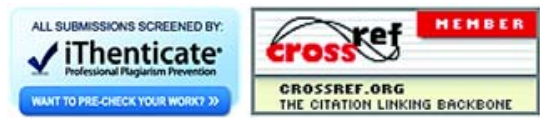


